\title{
The NUSTAR Project at FAIR
}

\section{Thomas Nilsson for the NUSTAR collaboration}

Fundamental Physics, Chalmers University of Technology, S-41296 Gothenburg, Sweden

E-mail: thomas.nilsson@chalmers.se

\begin{abstract}
The FAIR facility, under construction at the GSI site in Darmstadt, will be addressing a wealth of outstanding questions within the realm of subatomic, atomic, plasma, bio-physics and applications through a combination of novel accelerators, storage rings and innovative experimental set-ups. One of the key installations is the fragment separator Super-FRS that will be able to deliver an unprecedented range of radioactive ion beams (RIBs) in the energy range of $0-1.5 \mathrm{GeV} / \mathrm{u}$. These beams will be distributed to three branches, each with its unique domain with respect to beam energies and properties. The highenergy branch will permit reactions with radioactive beams at relativistic energies, whereas the low-energy branch will supply decelerated beams for high-resolution spectroscopy, traps and laser spectroscopy. Finally, the ring branch will uniquely permit stored and cooled exotic beams for a range of methods only possible in a storage ring. Thus, by developing experimental set-ups tailored for these beams, there are several complementary possibilities to gain information on key nuclei and reaction, to further our understanding on contemporary questions within nuclear structure and nuclear astrophysics. This ambitious programme is to be exploited within the NUSTAR (Nuclear Structure, Astrophysics and Reactions) collaboration.
\end{abstract}

PACS numbers: $21,24,26,29.38$.-c

Keywords: FAIR, NUSTAR, radioactive beams, experimental set-up 


\section{Introduction}

The FAIR (Facility for Antiproton and Ion Beams) facility, currently under construction at the GSI site in Darmstadt, Germany will constitute one of the largest accelerator-based research infrastructures world-wide. Within an international effort, a complex of novel accelerators, storage rings and innovative experimental set-ups will be constructed in order to address front-line research questions within subatomic, atomic, plasma, bio-physics and applications. The envisaged research is to be conducted within four experimental pillars, addressing hadron physics (PANDA[1]), compressed baryonic matter in relativistic heavy-ion collisions (CBM[2]), atomic physics and applications (APPA 3] ) and nuclear structure, reactions and astrophysics using radioactive beams (NUSTAR [4, 5]).

FAIR will be constructed in stages, starting with the so-called modularized start version (MSV) 6] with the ultimate goal to reach the full facility as described in [7. For an up-to-date overview of the FAIR facility, status and time-line of the construction, see [8].

Within the global landscape of RIB facilities [9], FAIR-NUSTAR will be the premiere European inflight facility [10. It will deliver the world-wide most energetic secondary beams and has the inherent advantage of "chemical blindness" compared to ISOLinstallations, i.e. all elements can be produced in the same manner. Consequently, a broad experimental programme utilising these beams is envisaged, under the umbrella of the NUSTAR collaboration assembling more than 800 members. The scientific questions to be addressed within the NUSTAR sub-projects have fundamental bearing on our understanding of the subatomic world and the inner workings of our universe. In contrast to many other scientific disciplines, there are not only a few, critical issues to be studied but rather a number of highly relevant and interrelated questions. A few examples would be:

- How are complex nuclei built from their basic constituents?

- What is the effective nucleon-nucleon interaction?

- How does QCD constrain its parameters?

- What are the limits for existence of nuclei?

- Where are the proton and neutron drip lines situated?

- Which new structures arise near or at the limits?

- How does the nuclear force depend on varying proton-to-neutron ratios?

- What is the isospin dependence of the spinorbit force?
- How does shell structure change far away from stability?

- How to explain collective phenomena from individual motion?

- What are the phases, relevant degrees of freedom, and symmetries of the nuclear many-body system?

- Which are the nuclei relevant for astrophysics and what are their properties?

- What is the origin of the heavy elements?

Any attempt of addressing the above issues is completely dependant on Radioactive Ion Beams (RIBs), covering a variety of energies and nuclear species, as well as state-of-the art instrumentation. Thus, these goals are common for many existing and future RIB facilities world-wide.

\section{Accelerator infrastructure and radioactive beam production with the Super-FRS}

FAIR builds on the existing accelerator infrastructure at GSI 11 and will rely on the UNILAC and SIS18 as first stages in the injector chain, following upgrades of these systems. The synchrotron SIS100 and a proton LINAC will be added in order to increase the energy for all beams and make production of antiprotons possible. In particular, SIS100 will be able to deliver primary beams of $10^{12}{ }^{238} \mathrm{U}^{28+}$ at $1.5-2 \mathrm{GeV} / \mathrm{u}$, corresponding to an increase in intensity of 2 to 3 orders of magnitude with respect to the current GSI synchrotron SIS18. It is followed by the Super-FRS for radioactive beam production, target stations, antiproton separator and the CR and HESR storage rings.

The Super-FRS 12 is a superconducting fragment separator that will constitute a crucial and integral part of every experiment using radioactive beams (RIBs) at FAIR. As can be seen in fig. 1, the device consists of a pre-separator (using radiation-hard normal-conducting magnets) and a main separator (using superconducting magnets). The large-aperture design of the elements yields an unprecedented acceptance which, e.g. for production of fission fragments having a large kinetic energy in the centre-of-mass system, will increase the transmission greatly compared to the existing FRS. Combined with the increased primary beam intensity, up to a factor $10^{4}$ improvement in RIB intensity can be expected, furthermore, also the heaviest exotic nuclei can be accessed and identified without charge-state ambiguities at these energies.

The Super-FRS feeds three branches, each having specific beam properties and associated experimental set-ups. The low-energy branch (LEB) houses experiments using energy-degraded beams for intermediateenergy reactions and decay studies with stopped 


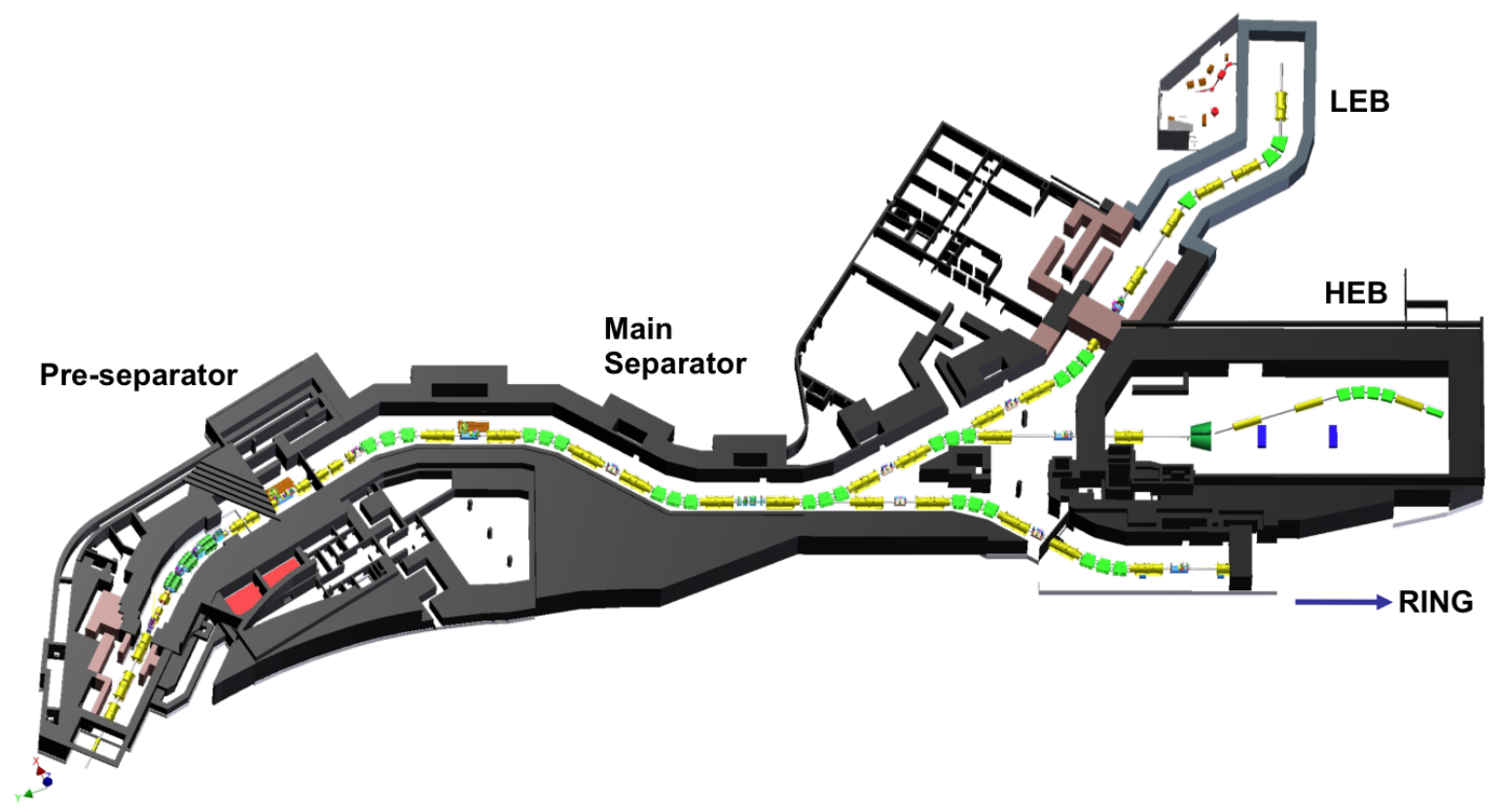

Figure 1. Layout of the Super-FRS with pre-separator, main separator and its three branches, low-(LEB), high-energy(HEB) and ring branch. The LEB hall shown is not included in the MSV.

beams, planned in the HISPEC and DESPEC projects. By combining with a gas-filled stopping cell [13] coupled to an MR-TOF mass filter, low-energy RIBs otherwise restricted to ISOL-type facilities can be achieved. Here, laser spectroscopy and Penning trap mass measurements will be performed in the LaSPEC and MATS experiments. The MSV foresees only an inadequate experimental hall for the majority of the activities of these four projects, thus a concerted action to fund and construct a building outside the startversion has been initiated by the NUSTAR collaboration. This includes a realistic time planning with milestones for secured funding, permissions etc. This building would as well house the energy buncher/spectrometer (inside the MSV) needed to efficiently degrade the energetic beams, as well as opening up the possibility for highresolution spectrometer experiment in a dispersionmatched design 14 .

The high-energy branch (HEB) takes the relativistic beam as produced from the Super-FRS and acts as such as a natural continuation of the device. The associated high-energy cave houses exclusively the $\mathrm{R}^{3} \mathrm{~B}[7$ experiment for reaction studies using relativistic radioactive beams. Finally, the ring branch feeds the CR (Collector Ring) with radioactive ions, followed by the NESR (New Experimental Storage Ring) in the full version of the FAIR facility, where the ILIMA[15] inring mass measurement, the EXL[16] (reactions at low momentum transfer) and the ELISe 17] (electron-ion collider) experiments are to be situated. The omission of the NESR in the MSV postpones the full realisation of those projects well into the next decade, however, parts of the ILIMA programme can be performed using the CR only.

\section{The NUSTAR projects within the Modularized Start Version - scope and status}

\section{1. $H I S P E C / D E S P E C$}

High-resolution reaction spectroscopy using intermediate energy RIBs as well as decay spectroscopy using stopped beams is the domain of the HISPEC and DESPEC projects. The precursor project PRESPEC 18, 19] has been running successfully for several years at GSI, in spite of very limited beam-time availability. Here, the HISPEC key systems LYCCA 20, and the AGATA 21] demonstrator for ion identification and gamma-ray detection, respectively have been employed. Further detection systems for neutrons, fast timing, total absorption spectroscopy etc are foreseen and well underway.

\subsection{MATS and LaSPEC}

The low-energy beams from the gas cell at the lowenergy branch will be used to investigate groundstate properties of exotic nuclei through atomic physics techniques 22, mass measurements in a Penning trap 
within the MATS project and laser spectroscopy using a variety of methods in the LaSPEC project. Details on MATS and LaSPEC can be found in the approved Technical Design Report 23]. The projects are already now in a very advanced state, having constructed the prototype TRIGA-SPEC 24, in operation at the TRIGA research reactor in Mainz.

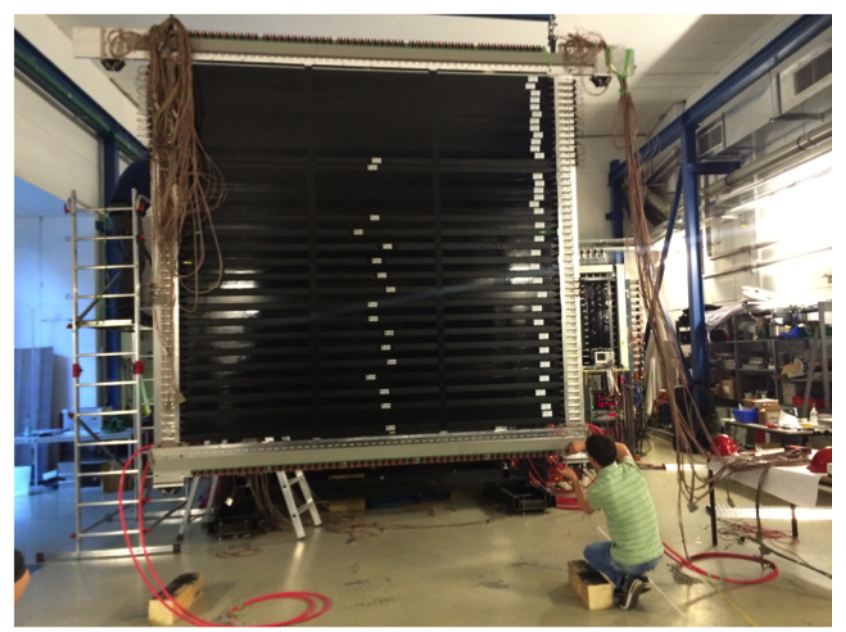

Figure 2. Part of the NeuLAND detector being assembled for beam tests at GSI.

\section{3. $R^{3} B$}

The $\mathrm{R}^{3} \mathrm{~B}$ (Reactions with Relativistic Radioactive Beams) project aims at complete kinematics detection of all messengers stemming from a very large range of high-energy reactions. Precursor experiments using the ALADIN/LAND setup at GSI have been successfully performed since more than two decades, however, the $\mathrm{R}^{3} \mathrm{~B}$ detection systems will be state-ofthe-art devices meaning a major step forward in terms of resolution and efficiency. The secondary reaction target will be surrounded by a silicon micro-vertex tracker, followed by the CALIFA 25] calorimeter for detection of gamma rays and light ions, projectile-like particles and fragments will be separated in the GLAD large-aperture superconducting dipole magnet 26] and subsequently detected in the NeuLAND 27 detector for high-energy neutrons as well as tracking and timeof-flight detectors for protons and heavy ions. As an option, complementary to the low-momentum transfer experiments foreseen for EXL, an active target can replace the target spectrometer.

Parts of the final CALIFA, NeuLAND and microvertex tracker systems have already been constructed and tested with beams at GSI, as shown in fig. 2. Since the GLAD magnet will be delivered early 2015 to GSI, the full $R^{3} B$ set-up can be assembled and tested in the existing GSI cave, before moving to the high-energy cave after completion. This will permit commissioning and pilot experiments well ahead of the first beams from Super-FRS become available.

\subsection{ILIMA}

In-ring mass measurements and decay studies of exotic isotopes is a long-standing activity that has been pursued in the ESR storage ring, see e.g. 28] for a recent overview, and constitutes the basis for the ILIMA project at FAIR. The CR will be a largeacceptance storage ring employing stochastic cooling and is thus suitable for mass measurements using isochronous beams, complemented with ToF detection of individual ions. This method is excellently suited for short-lived nuclides and will, in conjunction with the high RIB intensities available from the Super-FRS, make direct mass measurements of very exotic nuclei possible, including several that are expected to be rprocess waiting points.

\subsection{Additional experiments}

The Super-FRS itself constitutes a device that can be utilised for specific experiments, both for e.g. exploratory rare isotope yield studies as well as a highresolution spectrometer. Examples using the latter mode would be studies of mesic nuclei or probing the tensor interaction 29 via $(p, d)$-reactions. The Super-FRS physics collaboration has recently been formed and its scientific programme is currently being evaluated 30. by the FAIR management and scientific bodies.

Another activity being considered to be joined to the NUSTAR collaboration is the SHE (Superheavy Element) collaboration [31, with focus on synthesis, nuclear structure, atomic physics, and nuclear chemistry experiments with elements Z $\geq 104$. Development work for a high-intensity cw-Linac is ongoing, this would benefit the future of SHE research greatly.

\section{Prospects for NUSTAR physics using storage rings in the MSV and beyond}

The combination of the pulsed beam available from the FAIR synchrotrons with in-flight production and separation of RIBs is the optimal prerequisite for nuclear physics with storage rings 32. In spite of this fact, the staging of FAIR has in particular impacted the storage ring part of the NUSTAR programme through the delayed construction of the NESR, shown in fig. 4. This obstructs the EXL and ELISe projects, both due to lack of space in the CR and the absence of electron cooling needed for the stochastically precooled beams. Also the ILIMA project is curtailed by the omitted NESR, in particular the decay studies and high-precision Schottky mass spectrometry. 


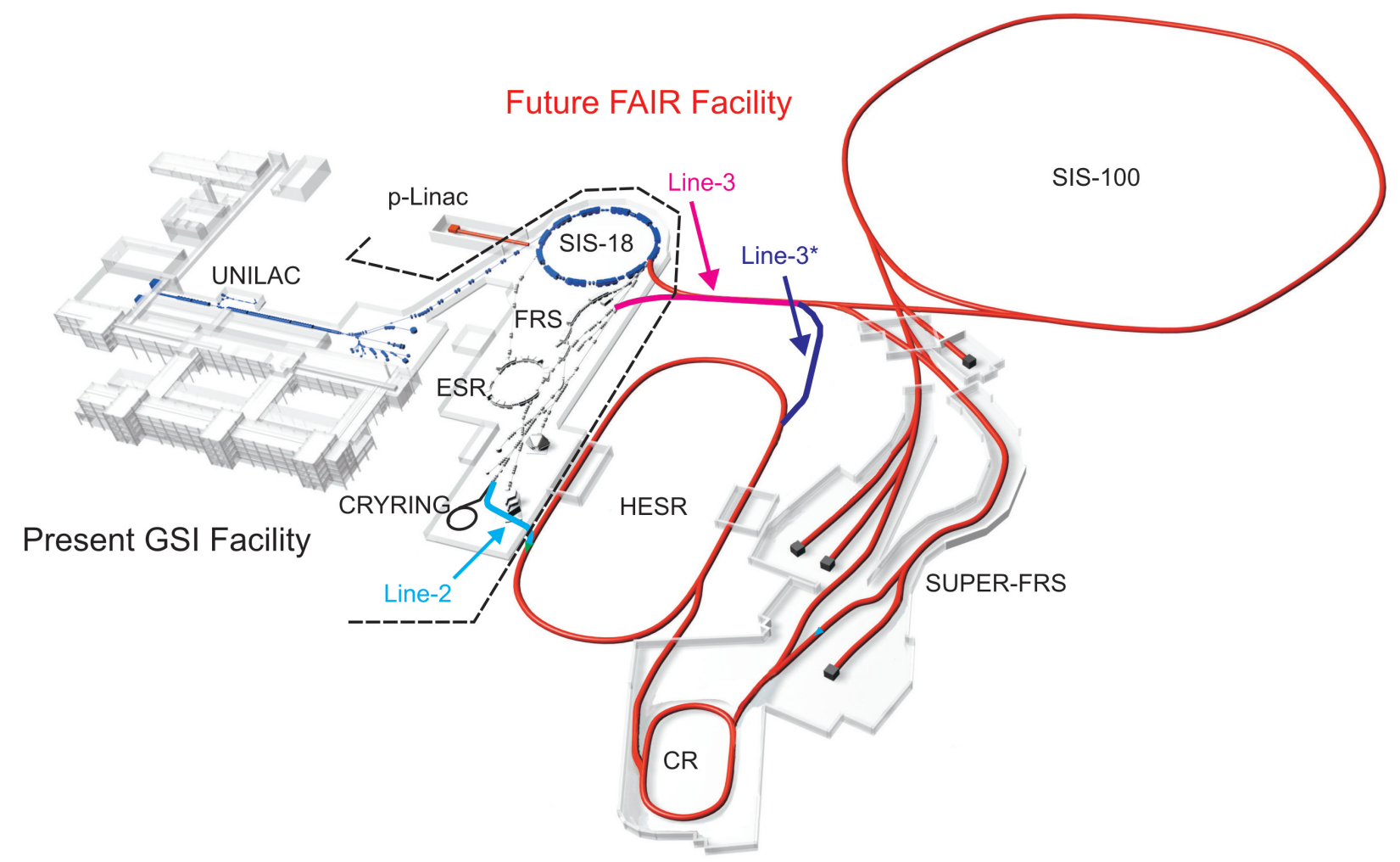

Figure 3. Layout of the FAIR MSV infrastructure showing possible additions (Line-2,3,3*) permitting to perform parts of the NUSTAR storage rings programme in the HESR and/or the existing ESR. Line-2 would permit an early injection of ions from the existing SIS-FRS-ESR complex into the HESR, ahead of completing the construction of the MSV. Line-3 + Line-3* would allow for transporting cooled beams of cooled RIBs and antiprotons to the ESR. The position of CRYRING, currently being reconstructed, is as well indicated. Courtesy of Yu. A. Litvinov.

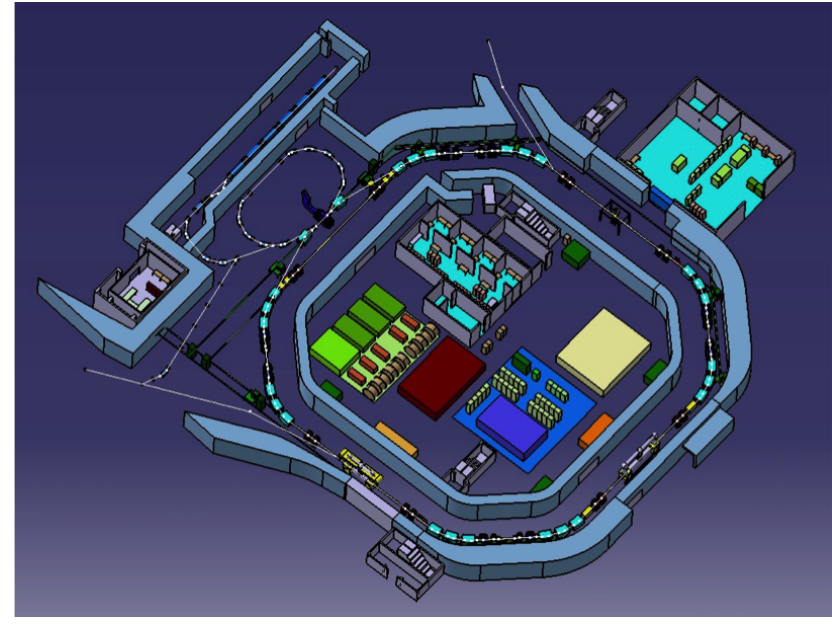

Figure 4. NESR layout including the electron LINAC and collider ring for ELISe as foreseen for the full FAIR project.
Nevertheless, the delay of the NESR has triggered intense activities in order to realise parts of the here envisaged programme in the storage rings contained in the MSV and/or already existing at GSI (see e.g. 33 for opportunities explored by the APPA-SPARC collaboration), and a joint NUSTAR-APPA Storage Ring Task Force was formed in 2013 in order to explore these opportunities further. As previously mentioned, the part of the ILIMA programme focused on nuclei with the very shortest half-lives can be pursued using the $\mathrm{CR}$ in isochronous mode. Furthermore, lifetime measurements of longer-lived nuclei could be done by subsequent injection and measurements in the HESR. By adding a return transfer line (depicted as Line$3^{*}$ in figure 33 from HESR to the existing installation at GSI, cooled beams of both radioactive beams and antiprotons could be made available in the ESR at rigidities up to $9.5 \mathrm{Tm}$ and, through deceleration and subsequent transfer, as well at low energies $(\leq 1.44 \mathrm{Tm})$ in CRYRING 34].

Having the RIBs of unprecedented intensity from 
the Super-FRS available in the ESR would open for an intermediate programme on low-momentum transfer reactions within the EXL project. Here, inring reactions on a gas-jet target combined with at complex detector set-up for charged-particle detection is envisaged. A proof-of-principle nuclear reaction experiment using stored beams in ESR was recently performed 35], using a newly developed solution to use DSSSDs as an active vacuum barrier towards the UHV regions of the storage ring 36 . By rearranging the lattice of the ESR, the sections available for experiments would increase and as well open up for an early implementation of the ELISe electron-ion collider 37, still within the boundaries of the existing building. The low energies and excellent vacuum conditions in CRYRING would permit studying key nuclear reactions with unstable beams for astrophysics 38, in addition to an envisaged rich programme within atomic physics and, in a longer perspective, lowenergy antiproton physics as planned in the FLAIR experiment. Nevertheless, the NESR would crucially be needed in the future to fully exploit the scientific opportunities at FAIR.

\section{Conclusions}

The NUSTAR programme at FAIR is well underway to take advantage of the beams from the Super-FRS as soon as they become available. The collaboration has made major steps forward in planning, securing funding and in many cases already constructing stateof-the-art detector systems, ensuring a rich scientific output from the very start. The alternative solutions for NUSTAR physics in storage rings will aid in securing this truly unique part of the programme also on a mid-term basis, training new generations of scientists while generating excellent physics output.

\section{Acknowledgements}

The author is indebted to numerous NUSTAR colleagues for providing information and material pertinent to this article. The Swedish Research Council is acknowledged for financial support.

\section{References}

[1] Bettoni D, Timmermans R and et al, eds. 2009 Physics Performance Report for: PANDA no. arxiv0903.3905v1

[2] Friman B, Höhne C, Knoll J, Leupold S, Randrup J, Rapp R and Senger P, eds. 2011 The CBM Physics Book vol. 814 of Lecture Notes in Physics (Springer Berlin Heidelberg)

[3] APPA collaboration 2012 Atomic and plasma physics, and applied sciences in bio, medical and material sciences (APPA) research pillar Tech. rep. http://www.flairatfair.eu/typo3/fileadmin/files/ documents/reports/APPA_SC_2012-11-13-final.pdf

[4] Rubio B and Nilsson T 2006 Nuclear Physics News 169
[5] Herlert A 2014 EPJ Web of Conferences $\mathbf{7 1}$

[6] 2009 The Modularized Start Version - a stepwise approach to the realization of FAIR Green paper FAIR

[7] Gutbrod H H, Augustin I, Eickhoff H, Groß K D, Henning W F, Krämer D and Walter G, eds. 2006 FAIR Baseline Technical Report (GSI)

[8] Lehmann I These proceedings

[9] Blumenfeld Y, Nilsson T and Van Duppen P 2013 Physica Scripta 2013014023

[10] Nilsson T 122013 Nucl. Instr. Meth. B 317194

[11] Gross K D 201411251993 Nuclear Physics News 314

[12] Geissel H et al. 52003 Nucl. Instr. Meth. B 20471

[13] Plaß W R et al. 122013 Nucl. Instr. Meth. B 317457

[14] Geissel H et al. 2013 Nucl. Instr. Meth. B 317277

[15] Walker P M, Litvinov Y A and Geissel H 92013 International Journal of Mass Spectrometry 349-350 247

[16] Egelhof P, Kisselev O, Münzenberg G, Neumaier S R and Weick H 2003 Physica Scripta 2003151

[17] Antonov A N et al. 52011 Nucl. Instr. Meth. A 63760

[18] Domingo-Pardo C, Bazzacco D, Doornenbal P, Farnea E, Gadea A, Gerl J and Wollersheim H J 122012 Nucl. Instr. Meth. A 694297

[19] Pietralla N et al. 2014 EPJ Web of Conferences 66

[20] Golubev P et al. 92013 Nucl. Instr. Meth. A $\mathbf{7 2 3} 55$

[21] Akkoyun S et al. 32012 Nucl. Instr. Meth. A 66826

[22] Blaum K, Dilling J and Nörtershäuser W 2013 Physica Scripta 2013014017

[23] Rodríguez D et al. 52010 Eur. Phys. J. Special Topics 183

[24] Ketelaer J et al. 92008 Nucl. Instr. Meth. A 594162

[25] Cortina-Gil D et al. 62014 Nuclear Data Sheets 12099

[26] Gastineau B et al. June 2010 Applied Superconductivity, IEEE Transactions on $\mathbf{2 0} 328$

[27] $\mathrm{R}^{3} \mathrm{~B}$ collaboration 2011 Technical report for the design, construction and commissioning of NeuLAND Tech. rep. http://www.fair-center.eu/fileadmin/fair/ experiments/NUSTAR/Pdf/TDRs/NeuLAND-TDR-Web.pdf

[28] Bosch F and Litvinov Y A 92013 International Journal of Mass Spectrometry 349-350 151

[29] Tanihata I 2013 Physica Scripta 2013014021

[30] Super-FRS Collaboration 2014 Scientific program of the Super-FRS collaboration: report of the collaboration to the FAIR management Tech. Rep. GSI Report 2014-4, doi:10.15120/GR-2014-4 GSI Darmstadt

[31] Düllmann C 2014 private communication

[32] Litvinov Y A et al. 122013 Nucl. Instr. Meth. B 317603

[33] Stöhlker T, Litvinov Y, Bräuning-Demian A, Lestinsky M, Herfurth F, Maier R, Prasuhn D, Schuch R and Steck M 2014 Hyperfine Interactions $\mathbf{2 2 7} 45$

[34] Lestinsky M These proceedings

[35] Kiselev $\mathrm{O}$ These proceedings

[36] Streicher B, Egelhof P, Ilieva S, Kalantar-Nayestanaki N, Kollmus H, Kröll T, Mutterer M, von Schmid M and Träger M 102011 Nucl. Instr. Meth. A 654604

[37] Simon H These proceedings

[38] Woods P These proceedings 